\title{
Erratum to: The Influence of Food Texture and Liquid Consistency Modification on Swallowing Physiology and Function: A Systematic Review
}

\author{
Catriona M. Steele - Woroud Abdulrahman Alsanei - Sona Ayanikalath • \\ Carly E. A. Barbon - Jianshe Chen · Julie A. Y. Cichero $\cdot$ Kim Coutts • \\ Roberto O. Dantas · Janice Duivestein - Lidia Giosa - Ben Hanson - Peter Lam • \\ Caroline Lecko • Chelsea Leigh $\cdot$ Ahmed Nagy • Ashwini M. Namasivayam • \\ Weslania V. Nascimento · Inge Odendaal · Christina H. Smith · Helen Wang \\ Published online: 3 March 2015 \\ (C) Springer Science+Business Media New York 2015
}

\section{Erratum to: Dysphagia}

DOI 10.1007/s00455-014-9578-x

In the original version of this article, Fig. 1 was published incorrectly. The corrected figure is given below.

The online version of the original article can be found under doi: 10 . 1007/s00455-014-9578-x.

C. M. Steele $\cdot$ J. Chen $\cdot$ J. A. Y. Cichero .

R. O. Dantas · J. Duivestein · B. Hanson - P. Lam - C. Lecko International Dysphagia Diet Standardisation Initiative (IDDSI)

Working Committee, Brisbane, Australia

e-mail: jschen@zjgsu.edu.cn

J. A. Y. Cichero

e-mail: Julie.cichero@iddsi.org

R. O. Dantas

e-mail: rodantas@fmrp.usp.br

J. Duivestein

e-mail: jduivestein@cw.bc.ca

B. Hanson

e-mail: ben.hanson@ucl.ac.uk

P. Lam

e-mail: peter.lam@iddsi.org

C. Lecko

e-mail: caroline.lecko@nhs.net

C. M. Steele $(\bowtie) \cdot$ C. E. A. Barbon · L. Giosa - C. Leigh ·

A. Nagy $\cdot$ A. M. Namasivayam $\cdot$ H. Wang

Toronto Rehabilitation Institute - University Health Network, 550

University Avenue, \#12-101, Toronto, ON M5G 2A2, Canada

e-mail: catriona.steele@uhn.ca

URL: http://www.iddsi.org
C. E. A. Barbon

e-mail: carly.barbon@uhn.ca

L. Giosa

e-mail: lidia.giosa@mail.utoronto.ca

C. Leigh

e-mail: leigh.chelsea@gmail.com

A. Nagy

e-mail: ahmed.nagy@uhn.ca

A. M. Namasivayam

e-mail: ashwini.namasivayam@uhn.ca

H. Wang

e-mail: helenyi.wang@gmail.com

C. M. Steele - C. E. A. Barbon - A. M. Namasivayam Department of Speech-Language Pathology, University of Toronto, Toronto, Canada

W. A. Alsanei

School of Food Science and Nutrition, University of Leeds, Leeds, UK

e-mail: woroooud@hotmail.com 


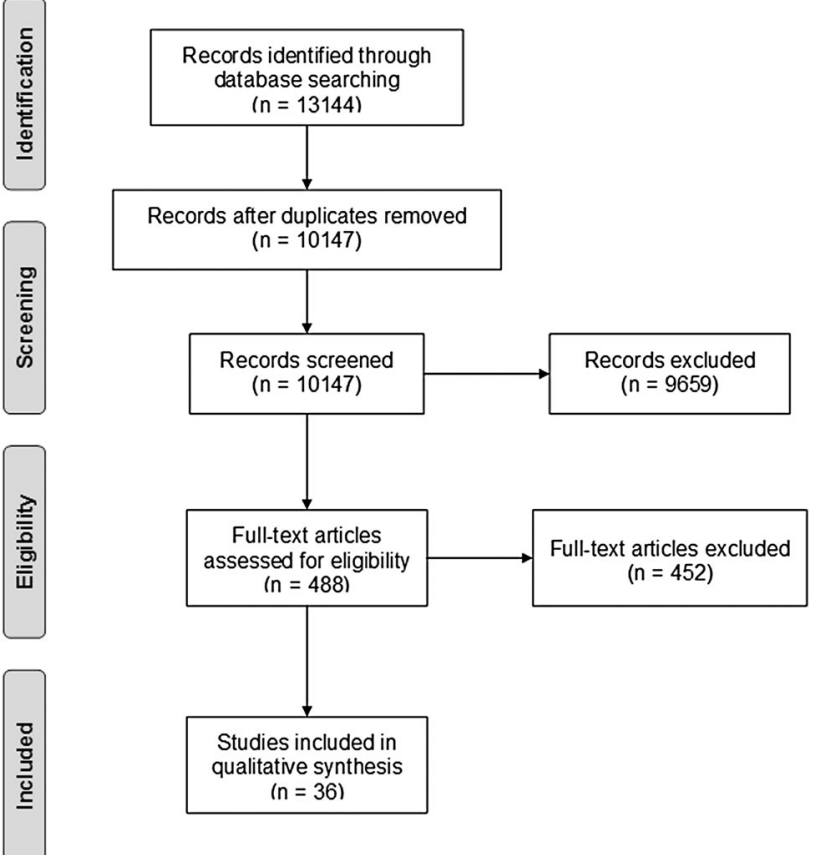

Fig. 1 PRISMA flow diagram of the search process used in this systematic review
Catriona M. Steele $\mathrm{PhD}$

Woroud Abdulrahman Alsanei $\mathrm{PhD}$ Cand

Sona Ayanikalath MSc

Carly E. A. Barbon MA

Jianshe Chen $\mathrm{PhD}$

Julie A. Y. Cichero $\mathrm{PhD}$

Kim Coutts MA

Roberto O. Dantas $\mathrm{PhD}, \mathrm{MD}$

Janice Duivestein MRSc, OT/PT

Lidia Giosa MHSc Cand

Ben Hanson $\mathrm{PhD}$

Peter Lam RD, CFE

Caroline Lecko RN

Chelsea Leigh BSc

Ahmed Nagy MSc, MD

Ashwini M. Namasivayam MHSc

Weslania V. Nascimento PhD Cand

Inge Odendaal BCommPath

Christina H. Smith $\mathrm{PhD}$

Helen Wang MHSc

\section{S. Ayanikalath}

Sheikh Khalifa Medical City, Abu Dhabi, United Arab Emirates e-mail: sonanarayanan4@gmail.com

J. Chen

Zhejiang Gongshang University, Hangzhou, China

J. A. Y. Cichero

School of Pharmacy, The University of Queensland, Brisbane, Australia

K. Coutts

Helen Joseph Hospital, Johannesburg, South Africa

e-mail: kim.coutts@gauteng.gov.za

R. O. Dantas · W. V. Nascimento

Medical School of Ribeirao Preto - University of Sao Paulo, Ribeirao Preto, Brazil

e-mail: fono.weslania@gmail.com

J. Duivestein

Sunny Hill Health Centre, Vancouver, BC, Canada

B. Hanson

Department of Mechanical Engineering, University College

London, London, UK
P. Lam

Peter Lam Consulting, Vancouver, BC, Canada

P. Lam

Faculty of Land and Food Systems, University of British

Columbia, Vancouver, BC, Canada

C. Lecko

National Health Service Commissioning Board Special Health Authority, London, UK

A. Nagy

Fayoum University, Fayoum, Egypt

I. Odendaal

University of KwaZulu-Natal, Durban, South Africa

e-mail: ingeoden@gmail.com

C. H. Smith

Division of Psychology and Language Sciences, University

College London, London, UK

e-mail: christina.smith@ucl.ac.uk 